\title{
AUTOMORPHIC FORMS ON NONDISCRETE MÖBIUS GROUPS
}

\author{
by A. F. BEARDON
}

(Received 18 January, 1995)

1. Introduction. If $\Gamma$ is a discrete Möbius group acting on the upper half-plane $\mathscr{H}$ of the complex plane, the quotient space $\mathscr{H} / \Gamma$ is a Riemann surface $\mathscr{R}$ and the automorphic functions on $\Gamma$ correspond to meromorphic functions on $\mathscr{R}$. If $\Gamma$ is a nondiscrete Möbius group acting on $\mathscr{H}$, then $\mathscr{H} / \Gamma$ is no longer a Riemann surface, and it is obvious that in this case there are no nonconstant automorphic functions on $\Gamma$. The situation for automorphic forms is quite different. Automorphic forms of integral dimension for a discrete group $\Gamma$ correspond to meromorphic differentials on $\mathscr{R}$, but even if $\Gamma$ is nondiscrete it may still support nontrivial automorphic forms. The problem of classifying those nondiscrete Möbius groups which act on $\mathscr{H}$ and which support nonconstant automorphic forms of arbitrary real dimension was raised and solved (rather indirectly) in [2] where, roughly speaking, function-theoretic methods are used to analyse all possible polynomial automorphic forms of integral dimension, and the results obtained then used to analyse the more general situation.

The purpose of this paper is to show how one can obtain stronger, more transparent results, with simpler, shorter, and more direct, proofs by analysing the groups rather than the forms. The pivotal step is the observation that each automorphic form on a group $\Gamma$ extends to an automorphic form on the closure of $\Gamma$ in $S L(2, \mathbb{R})$. If $\Gamma$ is not discrete, then its closure contains a one-parameter subgroup and it is easy to see that the one-parameter subgroups are the only nondiscrete groups that support nonconstant forms; this is the content of [2] (although not stated in these terms), but beyond this we also provide explicit descriptions of all possible automorphic forms.

2. Automorphic forms. Each element $\gamma$ of $S L(2, \mathbb{R})$ induces a Möbius map $g_{\gamma}: \mathscr{H} \rightarrow \mathscr{H}$ by the rule

$$
g_{\gamma}(z)=\frac{a z+b}{c z+d}, \quad \gamma=\left(\begin{array}{ll}
a & b \\
c & d
\end{array}\right),
$$

and in most situations one can change freely between $\gamma$ and $g_{\gamma}$. However, this freedom is not always available when considering automorphic forms or arbitrary real dimension for such forms have multipliers and, as pointed out by Rankin ([5], p. 72), a multiplier cannot always be transmitted from a matrix group to the corresponding Möbius group. Nevertheless, we shall continue to use the same symbol for both the matrix and the mapping (it is tiresome to do otherwise) but, as a consequence, we are obliged to maintain a clear distinction between matrices and maps. In particular, when we write $\gamma(z), \gamma(w), \ldots, \gamma$ denotes a Möbius map (and not a matrix).

With the matrix $\gamma$ given by (2.1), the function

$$
\mu(\gamma, z)=c z+d
$$

on $\operatorname{SL}(2, \mathbb{R}) \times \mathscr{H}$ satisfies the following elementary properties:

(a) $\mu(\alpha \beta, z)=\mu(\alpha, \beta(z)) \mu(\beta, z)$ (the Chain Rule);

Glasgow Math. J. 38 (1996) 249-253. 
(b) $\mu(I, z)=1, \mu(-I, z)=-1$;

(c) $\mu(\alpha,(-\beta)(z))=\mu(\alpha, \beta(z))$.

We shall need to consider $\mu(\gamma, z)^{k}$ for any real $k$. For each nonzero $w$, we define $w^{k}$ by

$$
w^{k}=\exp (\log w),
$$

where $\log (w)$ is the unique choice of the $\log$ arithm with $-\pi<\arg \omega \leq \pi$. Note that

(d) for all $w_{1}$ and $w_{2},\left|\left(w_{1} w_{2}\right)^{k}\right|=\left|w_{1}^{k}\right| \cdot\left|w_{2}^{k}\right|$, and

(e) for each real $k, \mu(\gamma, z)^{k}$ is meromorphic on $\mathscr{H}$.

if

A function $f$ is an automorphic form on $\Gamma$, of weight $k$ and multiplier system $v: \Gamma \rightarrow \mathbb{C}$

(1) $f$ is meromorphic on $\mathscr{H}$, and

(2) for all $z$ in $\mathscr{H}$, and all $\gamma$ in $\Gamma,|v(\gamma)|=1$ and

$$
f(\gamma(z))=v(\gamma) \mu(\gamma, z)^{k} f(z) \text {. }
$$

For an account of automorphic forms, see [3], [4] and [5]. We are using the terminology weight as in [5]; in [2] the same form $f$ has dimension $-k$.

There are various consistency conditions which must be satisfied by an automorphic form (which are sometimes needlessly included in the definition). Applying (2.2) to the matrices $\gamma, \eta$ and $\gamma \eta$, and assuming that $f$ is not identically zero, we obtain

$$
v(\gamma \eta) \mu(\gamma \eta, z)^{k}=v(\gamma) v(\eta) \mu(\gamma, \eta(z))^{k} \mu(\eta, z)^{k} .
$$

Putting $\gamma=I=\eta$, we find that $v(I)=1$. If $-I \in \Gamma$, we can also put $\gamma=-I$ in (2.2) and, because $f(z)=f((-I)(z))$, we see that $v(-I)=\exp (-i k \pi)$. This shows that $v(-I) \neq v(I)$ (unless $k$ is an even integer), so that for automorphic forms of nonintegral weight, the multiplier system $v$ on any $\Gamma$ that includes $-I$ does not project to a function on the quotient group $\Gamma /\{ \pm I\}$. This is why we must distinguish carefully between matrices and Möbius maps.

By (a) and (d) above,

$$
\left|\mu(\alpha \beta, z)^{k}\right|=\left|\mu(\alpha, \beta(z))^{k}\right|\left|\mu(\beta, z)^{k}\right|,
$$

so that by (e), the meromorphic function

$$
\sigma(\alpha, \beta)=\frac{\mu(\alpha, \beta(z))^{k} \mu(\beta, z)^{k}}{\mu(\alpha \beta, z)^{k}}
$$

is of unit modulus and so is independent of $z$. The expression $\sigma(\alpha, \beta)$ measures both the extent to which the Chain Rule fails for $k$-th powers of $\mu$, and also the extent to which $v$ fails to be a homomorphism for, rewriting (2.3) in terms of $\sigma$, we obtain

$$
v(\gamma \eta)=v(\gamma) v(\eta) \sigma(\gamma, \eta)
$$

We remark that (2.3) and (2.4) are (16) and (17) in [2]. Moreover, (2.5) can be used to give a very simple proof of Lemma 4 in [2].

It is well known that an automorphic form on $\Gamma$ induces a form of the same weight on the conjugate group $\phi^{-1} \Gamma \phi$ (see, for example, Lemma 3, [3], p. 276, and [4], p. 379). Briefly, with $\Gamma, f$ and $v$ as above, we define

$$
\Gamma^{*}=\phi^{-1} \Gamma \phi, \quad \gamma^{*}=\phi^{-1} \gamma \phi, \quad f^{*}(z)=\frac{f(\phi(z))}{\mu(\phi, z)^{k}}, \quad v^{*}\left(\gamma^{*}\right)=v(\gamma) \frac{\sigma(\gamma, \phi)}{\sigma\left(\phi, \gamma^{*}\right)} .
$$


Then, by straightforward computation (which we omit), we see that $f^{*}$ is an automorphic form, of weight $k$ and multiplier system $v^{*}$, on the group $\Gamma^{*}$. In fact, where necessary we may replace $\phi$ by $-\phi$; then $\left(f^{*}\right)^{*}=f$ so that the correspondence $f \mapsto f^{*}$ is a bijection between the forms for the two groups.

We end this section with the following crucial result already mentioned in $\$ 1$.

LEMMA 2.1. An automorphic form on a subgroup $\Gamma$ of $S L(2, \mathbb{R})$, of weight $k$ and multiplier system $v$, extends to an automorphic form on the closure $\Gamma^{c}$ of $\Gamma$.

Proof. Let $f$ be an automorphic form on $\Gamma$, of weight $k$ and with multiplier system $v$. Consider any $\gamma$ in $\Gamma^{c}$ and select $\gamma_{n}$ in $\Gamma$ converging to $\gamma$; then

$$
\left|\frac{f(\gamma(z))}{\mu(\gamma, z)^{k} f(z)}\right|=\lim _{n}\left|\frac{f\left(\gamma_{n}(z)\right)}{\mu\left(\gamma_{n}, z\right)^{k} f(z)}\right|=\lim _{n}\left|v\left(\gamma_{n}\right)\right|=1
$$

We now define

$$
v(\gamma)=\frac{f(\gamma(z))}{\mu(\gamma, z)^{k} f(z)}
$$

where the right hand side is a meromorphic function of $z$. By $(2.7),|v(\gamma)|=1$, so that $v(\gamma)$ is independent of $z$, and we see now that $f$ is an automorphic form on $\Gamma^{c}$ with multiplier $v$.

3. Automorphic forms on one-parameter subgroups of $S L(2, \mathbb{R})$. We begin by examining automorphic forms on the one-parameter group

$$
\mathscr{P}_{\infty}=\left\{\gamma_{t}: t \in \mathbb{R}\right\}, \quad \gamma_{t}=\left(\begin{array}{ll}
1 & t \\
0 & 1
\end{array}\right),
$$

consisting of all parabolic matrices with positive trace in $S L(2, \mathbb{R})$ that fix $\infty$. Suppose that $f$ is an automorphic form on $\mathscr{P}_{\infty}$. As $\mu\left(\gamma_{t}, z\right)=1,(2.2)$ and (2.3) imply that

$$
v\left(\gamma_{s+l}\right)=v\left(\gamma_{s} \gamma_{t}\right)=v\left(\gamma_{s}\right) v\left(\gamma_{t}\right), \quad f(z+t)=v\left(\gamma_{t}\right) f(z),
$$

and these two facts imply that $t \mapsto v\left(\gamma_{t}\right)$ is a continuous homomorphism of $\mathbb{R}$ into $S^{1}$. It is well known (and easy to show) that this implies that $v\left(\gamma_{t}\right)=\exp (i \rho t)$ for some real number $\rho$. We deduce that $f(z+t)=\exp (i \rho t) f(z)$; thus

$$
f^{\prime}(z)=\left.\frac{\partial}{\partial t} f(z+t)\right|_{t=0}=i \rho f(z),
$$

so that $f(z)=A \exp (i \rho z)$ for some real constant $A$. It is immediate that this function is an automorphic form of weight $k$ (for any $k$ ) on $\mathscr{P}_{\infty}$, and this proves the following result.

Proposition 3.1. A function $f$ is an automorphic form on $\mathscr{P}_{\infty}$, with multiplier system $v$, if and only if, for some real numbers $A$ and $\rho$,

$$
f(z)=A \exp (i \rho z), \quad v\left(\gamma_{t}\right)=\exp (i \rho t) .
$$

By appealing to the relation (2.6) between automorphic forms on conjugate groups, we obtain the following extension of Proposition 3.1. 
Proposition 3.2. Suppose that $\gamma$ is given by (2.1), and that $c \neq 0$. If $f$ is an automorphic form on $\gamma^{-1} \mathscr{P}_{\infty} \gamma$, of weight $k$ with some multiplier system $v$, then there are real numbers $A$ and $\rho$ such that

$$
f(z)=\frac{A}{(c z+d)^{k}} \exp \left(i \rho\left[\frac{a z+b}{c z+d}\right]\right) .
$$

Propositions 3.1 and 3.2 imply, for example, that if $G$ is a subgroup of $\operatorname{SL}(2, \mathbb{R})$ whose closure contains $\mathscr{P}_{\infty}$, and also some element $\gamma$ with $\gamma(\infty) \neq \infty$, then $G$ cannot support a nonconstant automorphic form $f$. Indeed, such an $f$ would have to be simultaneously of the forms (3.1) and (3.2) and this is impossible. It is this observation that lies at the heart of our classification of the nondiscrete groups that support automorphic forms.

A similar argument applies to the group

$$
\mathscr{K}_{0, \infty}=\left\{\gamma_{t}: t>0\right\}, \quad \gamma_{t}=\left(\begin{array}{cc}
t & 0 \\
0 & 1 / t
\end{array}\right)
$$

consisting of all hyperbolic matrices with positive trace in $S L(2, \mathbb{R})$ that fix 0 and $\infty$. In this case, if $f$ is an automorphic form on $\mathscr{H}_{0, \infty}$, then

$$
\mu\left(\gamma_{t}, z\right)^{k}=1 / t^{k}, \quad v\left(\gamma_{s t}\right)=v\left(\gamma_{s} \gamma_{t}\right)=v\left(\gamma_{s}\right) v\left(\gamma_{t}\right), \quad f\left(t^{2} z\right)=v\left(\gamma_{t}\right) f(z) .
$$

The map $x \mapsto \exp x$ followed by $t \mapsto v\left(\gamma_{t}\right)$ is a continuous homomorphism of $\mathbb{R}$ into $S^{1}$ so that, for some real number $\rho, v\left(\gamma_{t}\right)=\exp (i \rho \log t)$. We deduce that $f^{\prime}(z)=i \rho f(z) / 2 z$ and this yields the next result.

Proposition 3.3. A function $f$ is an automorphic form on $\mathscr{H}_{0, \infty}$, with multiplier system $v$, if and only if, for some real numbers $A$ and $\lambda$,

$$
f(z)=A \exp (i \lambda \log z), \quad v\left(\gamma_{t}\right)=\exp (i \rho \log t) .
$$

Clearly, this result extends to a result (analogous to Proposition 3.2) which gives the automorphic forms on the groups conjugate to $\mathscr{H}_{0, \infty}$; we omit the details.

Finally, the group $\mathscr{E}_{i}$ of elliptic matrices in $\operatorname{SL}(2, \mathbb{R})$ that fix $i$ and $-i$ is conjugate to the group of matrices $\mathscr{E}$ corresponding to Euclidean rotations of the unit disc (the group $\mathscr{C}$ is a subgroup of $S L(2, \mathbb{C})$, and this conjugacy is in $S L(2, \mathbb{C})$, but this does not affect the analysis). The method used above shows that any automorphic form $f$ on $\mathscr{E}$ satisfies $\left|f\left(e^{2 i t} z\right)\right|=|f(z)|$ for all real $t$. As $f$ is meromorphic near the origin, there is an integer $l$ such that $F(z)=z^{-l} f(z)$ is analytic near the origin with $F(0) \neq 0$, and as $|F(z)|=|F(w)|$ whenever $|z|=|w|$, the Maximum Modulus Theorem implies that $|F|$ has a local nonzero minimum at the origin. We deduce that $F$ must be constant; thus, for some constant $A$, $f(z)=A z^{l}$. This result can now be rewritten in terms of the original group $\mathscr{C}_{i}$, or in terms of any group conjugate to this, but again we leave the details to the reader.

4. Automorphic forms on closed connected subgroups of $S L(2, \mathbb{R})$. It is now an easy matter to complete our discussion and we only give the main steps in the argument. First, the results in $\$ 3$, together with the way automorphic forms on one group correspond to automorphic forms on a conjugate group, show that a function cannot be an automorphic form for two distinct one-parameter subgroups of $S L(2, \mathbb{R})$. 
Suppose now that $\Gamma$ is a nondiscrete subgroup of $S L(2, \mathbb{R})$ that supports a nonconstant automorphic form $f$. The form $f$ extends to a form on the closure $\Gamma^{c}$ of $\Gamma$, and this then restricts to a form on the component $\Gamma_{0}$ of $\Gamma^{c}$ that contains $I$. It is well known (from Lie group theory) that as $\Gamma$ is nondiscrete, $\Gamma_{0}$ contains a one-parameter group (see, for example, [1], p. 17, Lemma 2.28); thus, by the remarks in the previous paragraph, $\Gamma_{0}$ is itself a one-parameter group, and by the results in $\$ 3$, this determines the most general automorphic form on $\Gamma$.

We end with a geometric statement of our result.

THEOREM. Suppose that $G$ is a nondiscrete subgroup of $S L(2, \mathbb{R})$ and that $G$ supports a nonconstant automorphic form with some multiplier system. Then $G$ (when viewed as a Möbius group) is an elementary group in the sense of Kleinian groups.

\section{REFERENCES}

1. J. F. Adams, Lectures on Lie groups, (Univ. Chicago Press, 1969).

2. M. I. Knopp, Polynomial automorphic forms and nondiscontinuous groups, Trans. Amer. Math. Soc., 123 (1966), 506-520.

3. M. I. Knopp, On powers of the theta-function greater than the eighth, Acta Arith., 46 (1986), 271-286.

4. H. Petersson, Theorie der automorphen Formen beliebiger reeler Dimension und ihre Darstellung durch eine neue Art Poincarescher Reihen, Math. Ann., 103 (1930), 369-436.

5. R. A. Rankin, Modular forms and functions, (Cambridge Univ. Press, 1977).

D.P.M.M.S.

UNIVERSITY OF CAMBRIDGE

16 Mill Lane

Cambridge CB2 1RL

ENGLAND 